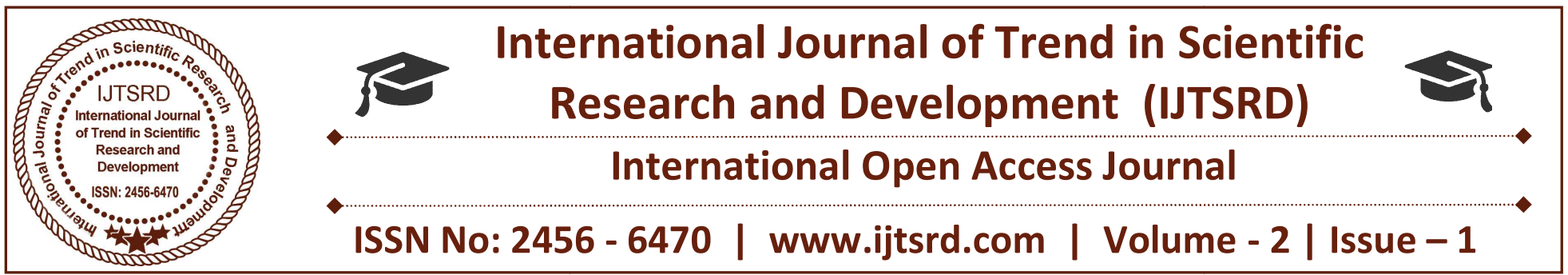

\title{
A Study of Incentive Scheme to allocate Big Data Tasks with Mobile Social Cloud
}

\author{
K. Gattaiah \\ M.Tech CSE, MCA, Lecturer in Computer Science \\ Govt.Degree College Wanaparthy, Telangana, India
}

\begin{abstract}
With the proliferation of increasingly powerful mobile devices, mobile users can collaboratively form a mobile cloud to provide pervasive services, such as data collecting, processing, and computing. With this mobile cloud, mobile crowdsourcing, as an emerging service paradigm, can enable mobile users to take over the outsourced tasks. By leveraging the sensing capabilities of mobile devices and integrating human intelligence and machine-computation, mobile crowdsourcing has the potential to revolutionize the approach of data collecting and processing. In this article we investigate the mobile crowdsourcing architecture and applications, and then discuss some research challenges and countermeasures for developing mobile crowdsourcing. Some research orientations are finally envisioned for further studies.
\end{abstract}

\section{INTRODUCTION}

According to the report of eMarketer in June 2014, the number of global smart phone users surpassed the one billion mark in 2012, and is estimated to be 1.75 billion in 2014. With the explosion of mobile devices, mobile computing has become an overwhelming trend in the development of IT technology as well as the fields of commerce and industry. However, mobile

Devices are facing some limitations on various resources, e.g., computation, memory, and energy. To overcome these limitations, mobile cloud computing has become a promising solution to enable mobile devices to consume varied cloud resources via wireless networks. Such a cloud computing service model, i.e. mobile as a service consumer (MaaSC), can improve the computation capability and energy efficiency of mobile devices by offloading computation tasks onto cloud servers [1]. New mobile devices are embedded with a set of versatile sensors, providing a novel paradigm to collect a vast amount of data about individuals, human society, and environments. Meanwhile, since mobile devices are usually associated with human users, humanintelligence can be leveraged for the tasks that are intractable for machine-computation, e.g., entity resolution and image annotation. Empowered by these capabilities, mobile devices shift from service consumers to service providers, offering a new service model for mobile cloud computing, i.e. mobile as a service provider (MaaSP). In this emerging service model, a large number of mobile devices connect with each other via wireless networks, forming an unprecedentedly powerful mobile cloud to provide pervasive data collecting, processing, and computing services. With this powerful mobile cloud, mobile crowdsourcing has been gaining momentum as a feasible solution for solving very large-scale problems. By outsourcing tasks to the mobile cloud, cost-effective and pervasive cloud services can be achieved, using a possibly huge number of mobile users and devices to work together in a distributed way. The ideas behind mobile crowdsourcing involve a wide range of applications and are utilized in different business models [2]. For example, OpenStreet Map [2] is a crowd sourced map of the world, created by worldwide voluntary mobile users using their local knowledge, GPS trajectories, and donated sources. The rapid development of OpenStreet Map indicates that mobile crowdsourcing has the potential to revolutionize traditional data processing and collecting approaches, and in fact already has. 


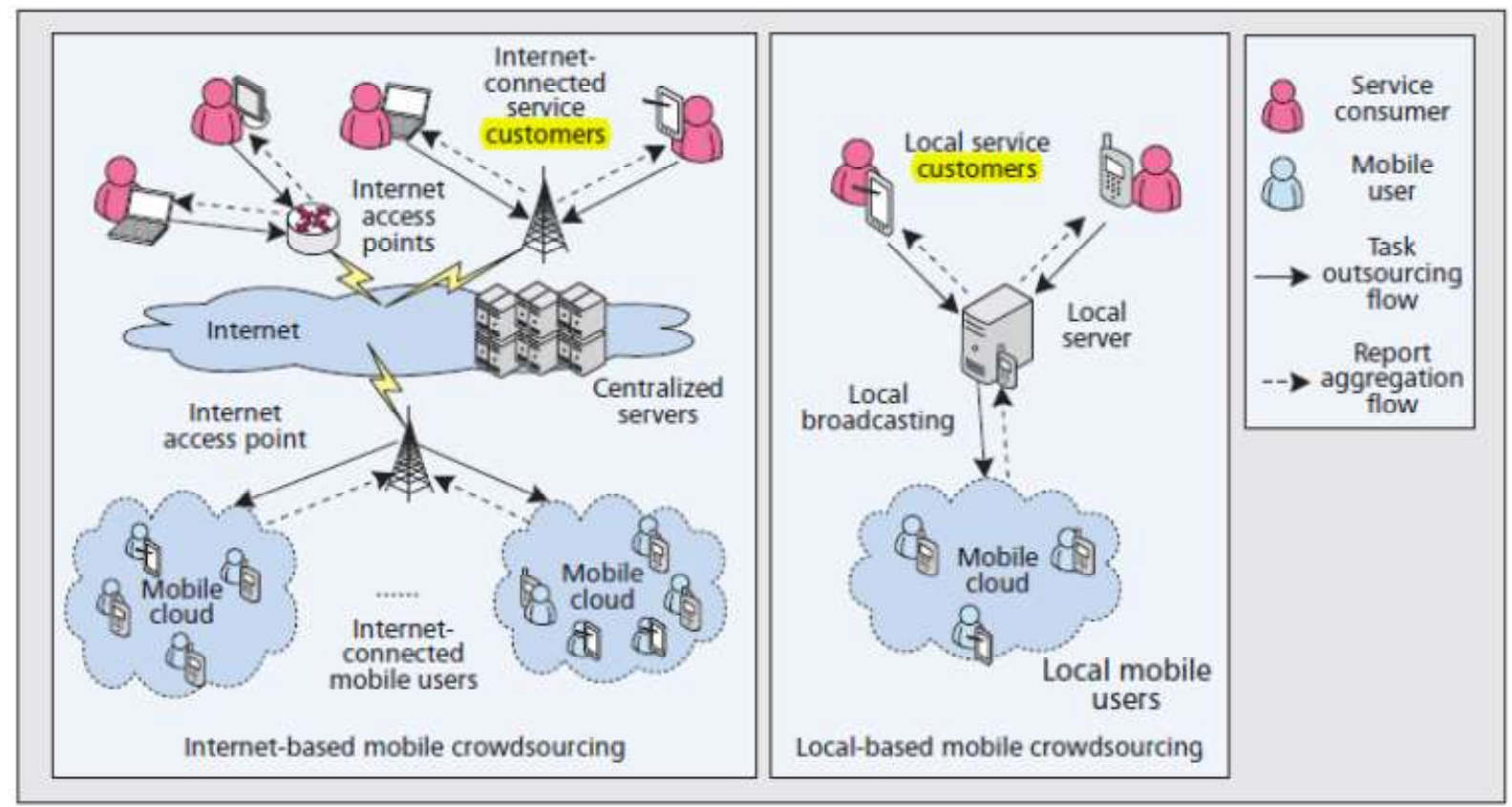

Figure 1.Network architectures of mobile crowdsourcing

II. MOBILE CROWDSOURCING: ARCHITECTURE AND APPLICATIONS MOBILE ARCHITECTURE

Mobile crowdsourcing is a type of electronic commerce service, where mobile users form a mobile cloud to sell cloud resources and services (e.g., data collecting, computing, and processing) for service consumers. Different from the traditional cloud computing that depends on Internet connection, mobile crowdsourcing can provide pervasive cloud services for both online and local terminals. Figure 1 shows the architectures of mobile crowdsourcing in an Internet-based scenario and a local-based scenario, respectively. The main difference between the two kinds of mobileCrowdsourcing models is that all the Internet connected mobile users can potentially be a service provider in the Internet-based mobile crowdsourcing, while only the mobile users in the vicinity can provide cloud services in local-based mobile crowdsourcing. We describe the key components of mobile crowdsourcing as follows.

Service Consumers: Service consumers refer to the online and local users that require cloud services through the mobile crowdsourcing system. They utilize the cloud services by outsourcing tasks to mobile users.

Mobile Users: Mobile users with mobile devices can autonomously form a mobile cloud to provide cloud services, for online service consumers via
cellular/WiFi networks, or for localservice consumers by communicating with local servers or neighboring users using Bluetooth/NFC techniques. When a mobile user participates in an outsourced task, it can adopt local computing or require mobile cloud computing to execute this task.

Centralized Servers: Centralized servers can be seen as a mobile crowdsourcing platform for Internet-based service consumers. They store all the crowdsourcing information (e.g., users' profiles, historical service records) that can be used for task outsourcing and service evaluation. Generally, centralized servers can provide trusted services for task publishing, allocating, report collecting, and feedback processing for the Internet-connected service consumers and mobile users.

Local Servers: Local servers can provide local crowdsourcing services, such as outsourced task broadcasting and task result aggregation, for service consumers and mobile users in the vicinity. Local servers are generally equipped with dedicated mobile local gateways to disseminate the task information to neighboring mobile users and collect user report results. In addition, they can also query or update necessary information from centralized servers to support mobile crowdsourcing. However, local servers are usually deployed for commercial purposes and not trusted by mobile users. 
III. MOBILE

APPLICATIONS

In the last five years mobile devices have become sensor and information hubs in our daily life. By integrating mobile computing and crowdsourcing, some emerging applications have shown the potential to achieve highly efficient and cost effective data computation, collection, and processing services. In this section we present two representative mobile crowdsourcing applications, as shown in Fig. 2:

\section{Mobile Crowdcomputing:}

Mobile crowdcomputing is used to outsource data computation tasks to mobile users. The mobile users who participate in the outsourced tasks can locally execute-

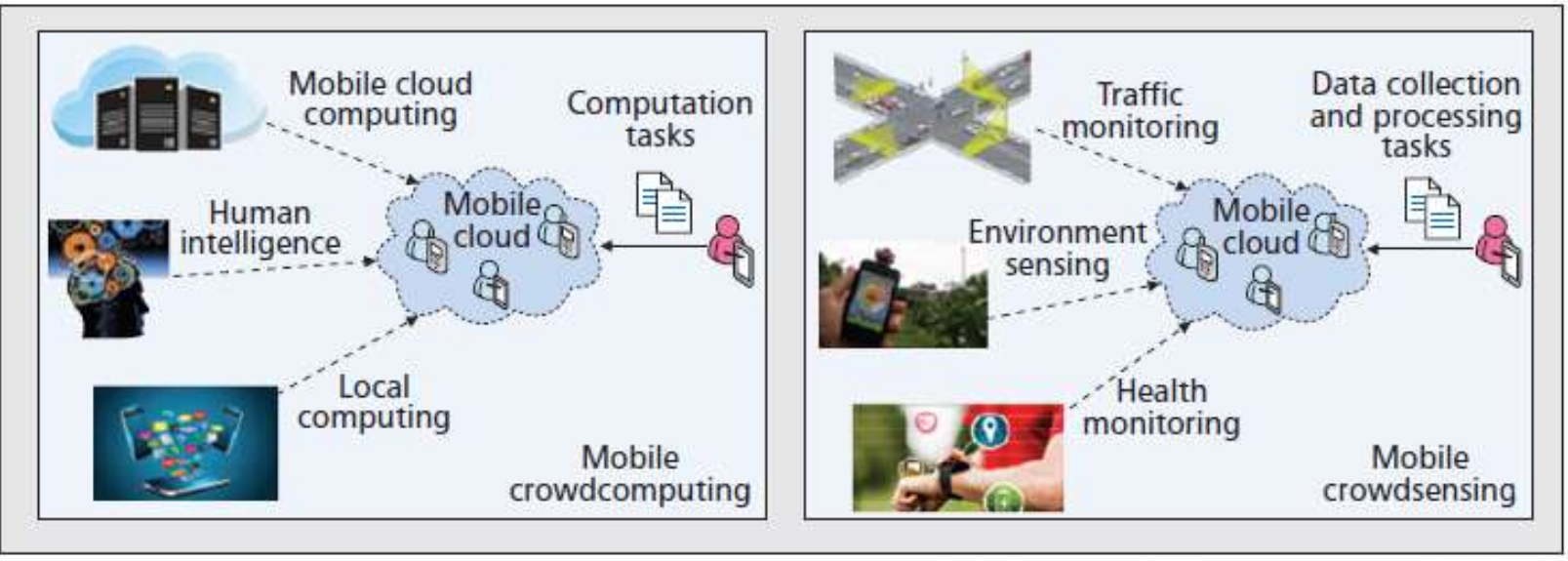

Figure 2.Mobile cloudcomputing and mobile crowdsensing

these tasks or offload them to the cloud severs based on their own data and computation resources. Due to human intervention, mobile crowdcomputing can leverage human-intelligence to deal with the tasks that are more suitable for human evaluation than machine computation (e.g., entity resolution, image annotation, and sentiment analysis). Honeybee [4] is a local-based mobile crowd computing application, in which face detection and photography tasks are outsourced to local mobile users. The mobile users use their mobile devices to run face detection algorithms and take specific photos, together with their personal evaluation. Crowd DB [3] crowdsources the computing tasks in the form of querying and answering, based on the Amazon Mechanical Turk platform.

\section{Mobile Crowdsensing:}

Data collection and processing, such as environment sensing and monitoring, generally require enormous technical efforts and significant economic resources. Mobile crowdsensing is used to outsource data collection and processing tasks to mobile users, who can perform data sensing with sensor-equipped mobile devices, and execute data processing by local computing or mobile cloud computing. By motivating mobile users' participation, mobile crowd sensing can provide cost-efficient mobile cloud services for data collection and processing. Signal Guru [5] is a localbased mobile crowdsensing application, utilizing
Smartphones to opportunistically detect current traffic signals and collaboratively exchange their detection information via an ad-hoc network. The smartphones can predict the future schedule of traffic signals based on the collection of exchanged information to guide the driving decision-making. Medusa [6] is a mobile crowd sensing application that collects specific sensing data by outsourcing sensing tasks, including video documentation, auditioning, and road monitoring, to Internet-connected mobile users via secure-HTTP based wireless communication.

\section{MOBILE}

CROWDSOURCING

\section{SELFISHNESS AND INCENTIVE}

Motivating mobile users to participate in mobile crowdsourcing is critical for forming a powerful Mobile cloud. The incentives to motivate mobile users could be varied, including financial rewards personal contribution, social gains, etc. Experiences with micro-task markets, such as Amazon Mechanical Turk, provide positive indications on monetary incentives, while Wikipedia is a good example of human contribution for non-financial gain. However, since both service consumers and mobile users are selfish and aim to benefit from crowdsourcing, incentive mechanisms should economically balance the requirements of the two parties, and create mutual benefits and a in-win situation. Propose two incentive mechanisms, including reward-sharing and auctionbased, to simulate mobile users to participate in 
mobile crowdsourcing. In reward sharing incentives, service consumer's offer fixed rewards for their outsourced tasks, and each reward is shared by the task participants according to the time they worked on the corresponding task. They also design a truthful auction based Incentive mechanism, where mobile users make offers for different outsourced tasks and service consumers choose appropriate participants to maximize their own utilities. Both the reward-sharing and auction-based incentive mechanisms can economically stimulate the formation of a mobile cloud and also can achieve mutual benefits.

\section{SECURITY THREATS}

Security is one of the primary concerns for cloud service consumers, while the mobile crowdsourcing philosophy originates from the assumption that mobile users would honestly provide accurate results. This is a contradiction and also a persistent problem for mobile crowdsourcing, since there may be malicious mobile users attempting to misbehave in or undermine the mobile crowdsourcing. The malicious users can fabricate computation or sensing results, or maliciously suspend the ongoing tasks, or launch other types of attacks that can directly or indirectly cause negative impacts on the outsourced tasks and service consumers. Some related work has been proposed to mitigate the impacts of malicious task reports and identify the misbehaving users. Develop a robust trajectory estimation strategy, to alleviate the negative influence of abnormal crowdsourced user trajectories and identify the normal and abnormal users.

\section{CONCLUSION}

In this article we have investigated the mobileCrowdsourcing architecture, and presented technical challenges with possible solutions to facilitate the implementation and development of

mobile crowdsourcing. By outsourcing tasks to mobile users, mobile crowdsourcing can provide a highly efficient and cost-effective way to achieve pervasive cloud services. We have also discussed future research directions and challenges to nurture continuous improvements for mobile crowdsourcing. It is envisioned that mobile crowdsourcing will accelerate the pervasiveness and evolution of data collecting, processing, and computing.

\section{REFERENCES}

1. D. Huang, T. Xing, and H. Wu, "Mobile Cloud Computing Service Models: A User-Centric Approach." IEEENetwork, vol. 27, no. 5, 2013, pp. 6-11.

2. A. Faggianiet al., "Smartphone-Based Crowdsourcing for Network Monitoring: Opportunities, Challenges, and a Case Study," IEEE Commun. Mag., vol. 52, no. 1, 2014, pp. 106-13.

3. J. Franklin et al., "Crowddb: Answering Queries with Crowdsourcing," Proc. ACM SIGMOD, 2011, pp. 61-72.

4. N. Fernando, W. Loke, and W. Rahayu, "Honeybee: A Programming Framework for Mobile Crowd Computing," Mobile and Ubiq. Syst.: Comp., Net., and Services, 2013, pp. 22436.

5. E. Koukoumidis, S. Peh, and M. R. Martonosi, "Signalguru: Leveraging Mobile Phones for Collaborative Traffic Signal Schedule Advisory," Proc. ACM MobiSys, 2011, pp. 127-40.

6. M. Ra et al., "Medusa: A Programming Framework for Crowd-Sensing Applications," Proc. ACM MobiSys, 2012, pp. 337-50.

7. D. Yang, G. Xue, X. Fang, and J. Tang, "Crowdsourcing to Smartphones: Incentive Mechanism Design for Mobile Phone Sensing," Proc. ACM Mobicom, 2012, pp. 173-84.

8. S. Reddy, D. Estrin, and M. Srivastava, "Recruitment Framework for Participatory Sensing Data Collections," Pervasive Comp., 2010, pp. 138-55.

9. S. He et al., "Toward Optimal Allocation of Location Dependent Tasks in Crowdsensing," Proc. IEEE INFOCOM, 2014, to appear. 